\title{
Performance Analysis for Analog Network Coding with Imperfect CSI in FDD Two Way Channels
}

\author{
Xidan PENG \\ Jiangxi Electric Power Corporation, Nanchang 330077, China \\ E-mail: sharon_ly_zhan@163.com \\ Xiangyang LI \\ Department of Electronic Engineering, Shanghai Jiao Tong University, Shanghai 200240, China \\ E-mail: lixiangyang@sjtu.edu.cn
}

\begin{abstract}
A time-division duplex (TDD) two-way channel exploits reciprocity to estimate the forward channel gain from the reverse link. Many previous works explore outage probabilities in the TDD system, based on the reciprocity property. However, a frequency-division duplex (FDD) system has no reciprocity property. In this letter, we investigate the impact of CSI estimation error on the performance of non-orthogonal and orthogonal analog network coding protocols in an FDD two-way system, where channel gains are independent of each other. Considering imperfect CSI, the closed-form expressions of outage probabilities by two protocols are derived in the high signal-to-noise ratio (SNR) regime, respectively. It is shown that the derived outage probabilities match results of Monte Carlo simulations in different communication scenarios. It is interesting that ANC in the FDD two-way channel is proved to outperform that in the TDD channel by the computer simulation.
\end{abstract}

Keywords two-way; ANC; imperfect CSI; FDD

\section{Introduction}

Cooperative relaying has been shown to be a practical technique to enhance the communication range of wireless networks. In some practical scenarios where data flows in both directions, a relay improves the performance of both transmission directions simultaneously. This pragmatic approach has been modeled as a two-way relay channel, and has recently attracted significant interests. Of particular interest is an analog network coding (ANC) protocol for this channel, which is a well-known amplify-and-forward (AF)-based two-way relaying protocol ${ }^{[1,2]}$. However, there works assume that receivers have the perfect knowledge of channel state information (CSI). With the impact of system noise and imperfect channel estimation algorithms, receivers hardly have the perfect knowledge of CSI. Therefore, performance analysis that take into account such CSI uncertainties play an important role in the design of practical systems.

Very recently, some related works on ANC have taken into consideration the impact of imperfect $\mathrm{CSI}^{[3-5]}$. These works consider a time division duplexing (TDD) system, where any given node exploits reciprocity to estimate the forward channel gain from the reverse link. This

Received October 28, 2014, accepted November 18, 2014 
condition can't be met in the FDD system since all channel gains are independent of each other. Thereby results of TDD systems in [3-5] aren't generated to FDD systems either due to more random variables. To the best of our knowledge, a performance analysis in term of outage probability in the FDD two way channel is still an open issue.

In this letter, we investigate the outage performance for ANC with imperfect CSI in the two way channel with FDD. Approximating expressions of outage probabilities for non-orthogonal and orthogonal ANC are derived in the high SNR regime. It is validated that Monte Carlo simulation results are in agreement with the derived outage probabilities.

\section{System Model}

In this letter, we consider an FDD two-way system with two sources $S_{1}, S_{2}$ and a relay $R$. Sources $S_{1}$ and $S_{2}$ desire to exchange their information with the help of relay $R$. We assume that all terminals are equipped with a single antenna and operate in a half-duplex mode. Suppose transmissions in this system suffer from frequency nonselective fading and additive noises. We denote channel gains from $S_{1}$ to $R, S_{2}$ to $R, R$ to $S_{1}$, and $R$ to $S_{2}$ by $h_{s_{1} r}, h_{s_{2} r}$, $h_{r s_{1}}$ and $h_{r s_{2}}$, respectively. They are modeled as zero-mean, circularly symmetric complex Gaussian random variables, denoted by $h_{s_{1} r} \sim \mathcal{C N}\left(0, \Omega_{h, s_{1} r}\right), h_{s_{2} r} \sim \mathcal{C N}\left(0, \Omega_{h, s_{2} r}\right), h_{r s_{1}} \sim$ $\mathcal{C N}\left(0, \Omega_{h, r s_{1}}\right)$ and $h_{r s_{2}} \sim \mathcal{C N}\left(0, \Omega_{h, r s_{2}}\right)$. These channel gains are assumed to be independent of each other, i.e. any given node cannot exploit reciprocity to estimate the forward channel gain from the reverse link in the FDD system. Considering channel estimation errors, we denote the estimations of channel gains by $\hat{h}_{s_{1} r}, \hat{h}_{s_{2} r}, \hat{h}_{r s_{1}}$ and $\hat{h}_{r s_{2}}$, respectively. We model the channel estimation by $h_{a b}=\hat{h}_{a b}+e_{a b}$, where $a, b \in\left\{s_{1}, s_{2}, r\right\}, \hat{h}_{a b} \sim \mathcal{C N}\left(0, \Omega_{\hat{h}, a b}\right), e_{a b} \sim \mathcal{C N}\left(0, \Omega_{e, a b}\right)$ is estimation error for channel gain $h_{a b}$. We also assume that all channel gains estimation errors are independent of each other. Without loss of generality, the transmit power of each terminal is set to be $P$, each additive noise power is $\Omega_{0}$. We also assume source node $S_{i}$ transmits with a fixed rate $v_{i}$ bits $/ \mathrm{Sec} / \mathrm{Hz}$.

\subsection{Non-orthogonal ANC}

Due to the half-duplex constraint and the complete separation between the two source nodes, the communication process of non-orthogonal ANC is organized into two transmission phases in Figure 1, where two different transmission phases work in different frequency bands.

\begin{tabular}{|c|c|c|c|}
\hline Protocol & The 1st transmission phase & The 2nd transmission phase & The 3rd transmission phase \\
\hline $\begin{array}{l}\text { Non- } \\
\text { orthogonal } \\
\text { ANC }\end{array}$ & & & \\
\hline $\begin{array}{l}\text { Orthogonal } \\
\text { ANC }\end{array}$ & & & \\
\hline
\end{tabular}

Figure 1 The two-way system where sources $S_{1}$ and $S_{2}$ desire to exchange their information via a relay $R$ 
In the first phase, both source nodes transmit simultaneously. The received signal at the relay $R$ is expressed as $Y_{r}=\sum_{i=1}^{2} h_{s_{i} r} \sqrt{P} X_{i}+n_{r}$, where $X_{i}$ is the unit energy transmit symbol from $S_{i}$ and $n_{r} \sim \mathcal{C N}\left(0, \Omega_{0}\right)$ is an additive noise at relay $R$. In the other phase, the relay amplifies its received symbol $Y_{r}$ and then broadcasts toward both source nodes. The received signal at the source node $S_{i}$ is given by $Y_{i}=h_{r s_{i}} \alpha_{n} Y_{r}+n_{i}$, where $\alpha_{n}$ is a power-scaled gain at relay $R$ and $n_{i} \sim \mathcal{C N}\left(0, \Omega_{0}\right)$ is an additive noise at source $S_{i}$. We consider the short-term power constraint at the relay node $R$, and thus the power-scaled gain, $\alpha_{n}$, is written as

$$
\alpha_{n}=\sqrt{\frac{P}{\left|\hat{h}_{s_{1} r}\right|^{2} P+\left|\hat{h}_{s_{2} r}\right|^{2} P+\Omega_{e, s_{1} r} P+\Omega_{e, s_{2} r} P+\Omega_{0}}}
$$

The signal $Y_{i}$ can be rewritten as

$$
\begin{aligned}
Y_{i}= & \hat{h}_{s_{j} r} \hat{h}_{r s_{i}} \alpha_{n} \sqrt{P} X_{j} \\
& +\hat{h}_{s_{i} r} \hat{h}_{r s_{j}} \alpha_{n} \sqrt{P} X_{i}(\text { Self-interference term }) \\
& +\left(\hat{h}_{s_{j} r} e_{r s_{i}} \alpha_{n}+\hat{h}_{r s_{i}} e_{s_{j} r} \alpha_{n}+e_{s_{j} r} e_{r s_{i}} \alpha_{n}\right) \sqrt{P} X_{j} \\
& +\left(\hat{h}_{s_{i} r} e_{r s_{i}} \alpha_{n}+\hat{h}_{r s_{i}} e_{s_{i} r} \alpha_{n}+e_{s_{i} r} e_{r s_{i}} \alpha_{n}\right) \sqrt{P} X_{i} \\
& +\hat{h}_{r s_{1}} n_{r} \alpha_{n}+e_{r s_{i}} n_{r} \alpha_{n}+n_{i}
\end{aligned}
$$

where $j$ is the other element in set $\mathcal{A}=\{1,2\}$, with a given element $i \in \mathcal{A}$ (e.g., if $i=1$, then $j=2$ ). Note that the definitions of $i$ and $j$ are used throughout this letter. Here we assume that the estimation of channel gains $\hat{h}_{s_{1} r}, \hat{h}_{s_{2} r}, \hat{h}_{r s_{i}}$ are obtained at source $S_{i}$ by using some channel estimation techniques. We assume both sources simultaneously transmit pilot symbols, and the relay amplifies and forwards the symbols to both sources. Source $S_{i}$ simultaneously estimates $\hat{h}_{s_{1} r}, \hat{h}_{s_{2} r}$ and $\hat{h}_{r s_{i}}$ by using the received pilot symbols (Alternatively, the relay estimates $\hat{h}_{s_{1} r}$ and $\hat{h}_{s_{2} r}$ by using pilot symbols, then forwards them to both sources ${ }^{[2]}$. Source $S_{i}$ also obtains $h_{r s_{i}}$ by using pilot symbols from the relay). The power scaled gain $\alpha_{n}$ is also derived according to (1). Therefore the self-interference term $\hat{h}_{s_{i} r} \hat{h}_{r s_{j}} \alpha_{n} \sqrt{P} X_{i}$ in (2) can be known and canceled at source $S_{i}$. Therefore, the mutual information per transmission from source $S_{j}$ to $S_{i}$ is given by

$$
\mathcal{I}_{s_{j} s_{i}}^{n}=\frac{1}{2} \log \left(1+\frac{\left|\hat{h}_{s_{j} r}\right|^{2}\left|\hat{h}_{r s_{i}}\right|^{2} P^{2}}{b_{1 i}\left(\left|\hat{h}_{s_{i} r}\right|^{2}+\left|\hat{h}_{s_{j} r}\right|^{2}\right)+b_{2 i}\left|\hat{h}_{r s_{i}}\right|^{2}+b_{3 i}}\right)
$$

where parameters $b_{1 i}, b_{2 i}$ and $b_{3 i}$ are denoted by

$$
\begin{aligned}
b_{1 i}= & \Omega_{e, r s_{i}} P^{2}+\Omega_{0} P \\
b_{2 i}= & \Omega_{e, s_{j} r} P^{2}+\Omega_{e, s_{i} r} P^{2}+\Omega_{0} P \\
b_{3 i}= & \Omega_{e, s_{j} r} \Omega_{e, r s_{i}} P^{2}+\Omega_{e, s_{i} r} \Omega_{e, r s_{i}} P^{2}+\Omega_{e, r s_{i}} \Omega_{0} P \\
& +\Omega_{e, s_{i} r} \Omega_{0} P+\Omega_{e, s_{j} r} \Omega_{0} P+\Omega_{0}^{2}
\end{aligned}
$$

\subsection{Orthogonal ANC}

The communication of Orthogonal ANC consists of three transmission phases in Figure 1. In the first two transmission phases, two sources transmit signal, respectively. When source $S_{j}$ transmits symbol $X_{j}$, signal $Y_{s_{j} r}$ overheard at relay $R$ and signal $Y_{s_{j} s_{i}}$ at source $S_{i}$ are 
expressed as

$$
\begin{aligned}
& Y_{s_{j} r}=\left(\hat{h}_{s_{j} r}+e_{s_{j} r}\right) \sqrt{P} X_{j}+n_{j r} \\
& Y_{s_{j} s_{i}}=\left(\hat{h}_{s_{j} s_{i}}+e_{s_{j} s_{i}}\right) \sqrt{P} X_{j}+n_{j i}
\end{aligned}
$$

where $n_{j r} \sim \mathcal{C N}\left(0, \Omega_{0}\right)$ and $n_{j i} \sim \mathcal{C N}\left(0, \Omega_{0}\right)$ are additive noises at relay $R$ and source $S_{i}$, respectively. In the third transmission phase, relay $R$ amplifies sum of signals $Y_{s_{1} r}$ and $Y_{s_{2} r}$, and forwards two sources. Source $S_{i}$ receives

$$
\begin{aligned}
Y_{r s_{i}}= & \hat{h}_{s_{j} r} \hat{h}_{r s_{i}} \alpha_{o} \sqrt{P} X_{j} \\
& +\hat{h}_{s_{i} r} \hat{h}_{r s_{i}} \alpha_{o} \sqrt{P} X_{i}(\text { Self-interference term }) \\
& +\left(\hat{h}_{s_{j} r} e_{r s_{i}} \alpha_{o}+\hat{h}_{r s_{i}} e_{s_{j} r} \alpha_{o}+e_{r s_{i}} e_{s_{j} r} \alpha_{o}\right) \sqrt{P} X_{j} \\
& +\left(\hat{h}_{s_{i} r} e_{r s_{i}} \alpha_{o}+\hat{h}_{r s_{i}} e_{s_{i} r} \alpha_{o}+e_{r s_{i}} e_{s_{i} r} \alpha_{o}\right) \sqrt{P} X_{i} \\
& +\hat{h}_{r s_{i}} n_{i r} \alpha_{o}+\hat{h}_{r s_{i}} n_{j r} \alpha_{o}+e_{r s_{i}} n_{i r} \alpha_{o}+e_{r s_{i}} n_{j r} \alpha_{o}+n_{i r}
\end{aligned}
$$

where $\alpha_{o}$ is the power scaled gain with the short-power constraint, denoted by

$$
\alpha_{o}=\sqrt{\frac{P}{\left|\hat{h}_{s_{1} r}\right|^{2} P+\left|\hat{h}_{s_{2} r}\right|^{2} P+\Omega_{e, s_{1} r} P+\Omega_{e, s_{2} r} P+2 \Omega_{0}}}
$$

Similar to non-orthogonal ANC, the self-interference term can be canceled at source $S_{i}$ to obtain residual signal $Y_{r s_{i}}^{c}$. Jointly proceeding signals $Y_{s_{j} s_{i}}$ and $Y_{r s_{i}}^{c}$ with the maximum combine ratio technique, orthogonal ANC achieves the mutual information per transmission as

$$
\begin{aligned}
\mathcal{I}_{s_{j} s_{i}}^{o}=\frac{1}{3} \log ( & +\frac{\left|\hat{h}_{s_{j} s_{i}}\right|^{2} P}{\Omega_{e, s_{j} s_{i}} P+\Omega_{0}} \\
& \left.+\frac{\left|\hat{h}_{s_{j} r}\right|^{2}\left|\hat{h}_{r s_{i}}\right|^{2} P^{2}}{c_{1 i}\left(\left|\hat{h}_{s_{i} r}\right|^{2}+\left|\hat{h}_{s_{j} r}\right|^{2}\right)+c_{2 i} \hat{h}_{r s_{i}}+c_{3 i}}\right)
\end{aligned}
$$

where parameters $c_{1 i}, c_{2 i}$ and $c_{3 i}$ are shown as

$$
\begin{aligned}
c_{1 i}= & \Omega_{e, r s_{i}} P^{2}+\Omega_{0} P, \\
c_{2 i}= & \Omega_{e, s_{j} r} P^{2}+\Omega_{e, s_{i} r} P^{2}+2 \Omega_{0} P, \\
c_{3 i}= & \Omega_{e, s_{j} r} \Omega_{e, r s_{i}} P^{2}+\Omega_{e, s_{i} r} \Omega_{e, r s_{i}} P^{2}+2 \Omega_{e, r s_{i}} \Omega_{0} P \\
& +\Omega_{e, s_{i} r} \Omega_{0} P+\Omega_{e, s_{j} r} \Omega_{0} P+2 \Omega_{0}^{2}
\end{aligned}
$$

\section{Outage Probability Analysis}

As clearly shown in (3) and (8), the forms of mutual information per transmission are not easily tractable due to the existence of imperfect channel gains and thus we approximately analyze their outage probabilities.

We define the system suffers from an outage if there exists one of both source nodes which decodes its received signals with error, and denote outage probabilities achieved by non-orthogonal ANC and orthogonal ANC by $\mathcal{P}_{\text {out }}^{n}$ and $\mathcal{P}_{\text {out }}^{o}$, respectively. The outage probability is expressed as

$$
\mathcal{P}_{\text {out }}^{z} \triangleq 1-\operatorname{Pr}\left(\bigcap_{i=1,2} \mathcal{I}_{s_{j} s_{i}}^{z} \geq v_{j}\right)
$$


where $x \in\{o, n\}$ and $v_{j}$ denotes the transmission rate of source node $S_{j}$. In the high SNR regime, system outage probability is approximated by its upper bound ${ }^{[6]}$, i.e.,

$$
\mathcal{P}_{\text {out }}^{z}=\sum_{i=1}^{2} \operatorname{Pr}\left(\mathcal{I}_{s_{j} s_{i}}^{z}<v_{j}\right)
$$

\subsection{Non-orthogonal ANC}

For convenience, we set parameters as follows

$$
\begin{array}{ll}
u=\frac{b_{1 i} \Omega_{0}}{b_{3 i} P}\left|\hat{h} s_{j} r\right|^{2} & v=\frac{b_{2 i} \Omega_{0}}{b_{3 i} P}\left|\hat{h} r s_{i}\right|^{2} \\
w=\frac{b_{1 i} \Omega_{0}}{b_{3 i} P}\left|\hat{h} s_{i} r\right|^{2} & \delta=\frac{\Omega_{0}}{P} \\
h(\delta)=\frac{\left(4^{v_{j}}-1\right) b_{1 i} b_{2 i}}{P^{2} b_{3 i}} \frac{\Omega_{0}}{P} &
\end{array}
$$

With these parameters and (3), probability $\operatorname{Pr}\left(\mathcal{I}_{s_{j} s_{i}}^{n}<v_{j}\right)$ can be expressed as

$$
\operatorname{Pr}\left(\mathcal{I}_{s_{j} s_{i}}^{n}<v_{j}\right)=\operatorname{Pr}\left(\frac{u v}{u+v+w+\delta}<h(\delta)\right)
$$

Theorem 1 If $h(\delta)$ is continuous with $h(\delta) \rightarrow 0$ as $\delta \rightarrow 0$, and variables $u, v, w$ are exponentially distributed with parameters $\lambda_{u}, \lambda_{v}, \lambda_{w}$ respectively, then

$$
\begin{aligned}
& \lim _{\delta \rightarrow 0} \frac{1}{h(\delta)} \operatorname{Pr}\left(\frac{u v}{u+v+w+\delta}<h(\delta)\right) \\
= & \lambda_{u}+\lambda_{v}-\left[c+\ln \left(\frac{\lambda_{u} \lambda_{v} h(\delta)}{\lambda_{w}}\right)\right] \frac{\lambda_{u} \lambda_{v}}{\lambda_{w}}
\end{aligned}
$$

where $c \approx 0.577$ is the Euler Lorenzo Mascheroni constant.

Proof Let $\mathcal{P}_{\mathrm{e}}=\operatorname{Pr}\left(\frac{u v}{u+v+w+\delta}<h(\delta)\right)$ and $x=u-h(\delta)$. The probability density function (PDF) of $x$ is written as

$$
f(x)= \begin{cases}\lambda_{u} \mathrm{e}^{-\lambda_{u}[x+h(\delta)]}, & x \geq-h(\delta) \\ 0, & x<-h(\delta)\end{cases}
$$

Then it is given by

$$
\begin{aligned}
\lim _{\delta \rightarrow 0} \frac{\mathcal{P}_{\mathrm{e}}}{h(\delta)}= & \lim _{\delta \rightarrow 0} \frac{1}{h(\delta)} \operatorname{Pr}[x \leq 0] \\
& +\lim _{\delta \rightarrow 0} \frac{1}{h(\delta)} \operatorname{Pr}\left[v<\frac{(u+w+\delta) h(\delta)}{x}, x>0\right] \\
= & \lim _{\delta \rightarrow 0} \frac{1}{h(\delta)}\left[1-\mathrm{e}^{-\lambda_{u} h(\delta)}\right]+g_{1}
\end{aligned}
$$

Setting $g_{2}=h(\delta)+\frac{[w+\delta+h(\delta)] h(\delta)}{x}, g_{3}=\lambda_{u} x+\frac{\delta h(\delta)+h^{2}(\delta)}{x}$, then

$$
\begin{aligned}
g_{1}=\lim _{\delta \rightarrow 0} \frac{1}{h(\delta)} \operatorname{Pr}\left[v<\frac{(u+w+\delta) h(\delta)}{x}, x>0\right] \\
=\lim _{\delta \rightarrow 0} \frac{1}{h(\delta)}\left[\int_{0}^{\infty} \int_{0}^{\infty}\left(1-\mathrm{e}^{-\lambda_{v} g_{2}}\right) f(x) f(w) \mathrm{d} x \mathrm{~d} w\right] \\
=\lim _{\delta \rightarrow 0} \frac{1}{h(\delta)}\left[\mathrm{e}^{-\lambda_{v} h(\delta)}-\lambda_{u} \mathrm{e}^{-\left(\lambda_{u}+\lambda_{v}\right) h(\delta)}\right. \\
\left.\times \int_{0}^{\infty}\left(\frac{\lambda_{w}}{\lambda_{w}+\lambda_{v} h(\delta) x^{-1}}\right) \mathrm{e}^{-g_{3}} \mathrm{~d} x\right]
\end{aligned}
$$


Let $\delta h(\delta)+h^{2}(\delta)=0$ due to $\delta h(\delta)+h^{2}(\delta)=\mathcal{O}\left(\delta^{2}\right)$. It becomes

$$
\begin{aligned}
\lim _{\delta \rightarrow 0} \frac{\mathcal{P}_{\mathrm{e}}}{h(\delta)}= & \lim _{\delta \rightarrow 0} \frac{1}{h(\delta)}\left[1-\mathrm{e}^{-\left(\lambda_{u}+\lambda_{v}\right) h(\delta)} \int_{0}^{\infty} \lambda_{u} \mathrm{e}^{-g_{3}} \mathrm{~d} x\right. \\
& \left.+\mathrm{e}^{-\left(\lambda_{u}+\lambda_{v}\right) h(\delta)} \int_{0}^{\infty}\left(\frac{\lambda_{u} \lambda_{v} h(\delta)}{\lambda_{w} x+\lambda_{v} h(\delta)}\right) \mathrm{e}^{-\lambda_{u} x} \mathrm{~d} x\right] \\
= & \underbrace{\lim _{\delta \rightarrow 0} \frac{1}{h(\delta)}\left[1-\mathrm{e}^{-\left(\lambda_{u}+\lambda_{v}\right) h(\delta)} \int_{0}^{\infty} \lambda_{u} \mathrm{e}^{-g_{3}} \mathrm{~d} x\right]}_{=\lambda_{u}+\lambda_{v} \text { (Please see Lemma 1.2 in [7]) }} \\
& +\int_{h(\delta) \rightarrow 0}^{\left.\infty \int_{\lambda_{u} \lambda_{v} \lambda_{w}^{-1} h(\delta)}^{\infty} \frac{1}{x} \mathrm{e}^{-x} \mathrm{~d} x\right]} \underbrace{\lambda_{w}}_{=E_{i}\left(\frac{\lambda_{u} \lambda_{v} h(\delta)}{\lambda_{w}}\right) \frac{\lambda_{u} \lambda_{v}}{\lambda_{w}} \text { (Please see Equation 4.331.2 in [9]) }}
\end{aligned}
$$

With Eq 4.331.2 in [9] and Lemma 1.2 in [7], it holds.

With (11), (13) and Theorem 1, probability $\mathcal{P}_{\text {out }}^{n}$ is derived in (19) in the high SNR regime $(\delta \rightarrow 0)$.

$$
\begin{aligned}
& \mathcal{P}_{\text {out }}^{n}= \sum_{i=1}^{2} \frac{\left(4^{v_{j}}-1\right) b_{1 i} b_{2 i} \Omega_{0}}{b_{3 i} P^{3}}\left[\frac{b_{3 i} P}{b_{1 i} \Omega_{0} \Omega_{\hat{h}, s_{j} r}}+\frac{b_{3 i} P}{b_{2 i} \Omega_{0} \Omega_{\hat{h}, r s_{i}}}\right. \\
&\left.\quad-\left(c+\ln \frac{\left(4^{v_{j}}-1\right) b_{1 i} \Omega_{\hat{h}, s_{i} r}}{P^{2} \Omega_{\hat{h}, s_{j} r} \Omega_{\hat{h}, r s_{i}}}\right) \frac{b_{3 i} P \Omega_{\hat{h}, s_{i} r}}{b_{2 i} \Omega_{0} \Omega_{\hat{h}, s_{j} r} \Omega_{\hat{h}, r s_{i}}}\right] \\
& \mathcal{P}_{\text {out }}^{o}= \sum_{i=1}^{2} \frac{\left(8^{v_{j}}-1\right)^{2} c_{1 i} c_{2 i} \Omega_{0}\left(\Omega_{e, s_{j} s_{i}} P+\Omega_{0}\right)}{2 c_{3 i} \Omega_{\hat{h}, s_{j} s_{i}} P^{4}} \\
& {\left[\frac{c_{3 i} P}{c_{1 i} \Omega_{0} \Omega_{\hat{h}, s_{j} r}}+\frac{c_{3 i} P}{c_{2 i} \Omega_{0} \Omega_{\hat{h}, r s_{i}}}-\left(c+\ln \frac{\left(8^{v_{j}}-1\right) c_{1 i} \Omega_{\hat{h}, s_{i} r}}{P^{2} \Omega_{\hat{h}, s_{j} r} \Omega_{\hat{h}, r s_{i}}}\right) \frac{c_{3 i} P \Omega_{\hat{h}, s_{i} r}}{c_{2 i} \Omega_{0} \Omega_{\hat{h}, s_{j} r} \Omega_{\hat{h}, r s_{i}}}\right] }
\end{aligned}
$$

\subsection{Orthogonal ANC}

Here some parameters are denoted by

$$
\begin{array}{lll}
x=\frac{c_{1 i} c_{2 i} \Omega_{0}\left|\hat{h}_{s_{j} s_{i}}\right|^{2}}{c_{3 i}\left(\Omega_{e, s_{j} s_{i}} P+\Omega_{0}\right) P^{2}}, & u=\frac{c_{1 i} \Omega_{0}}{c_{3 i} P}\left|\hat{h} s_{j} r\right|^{2} \\
v=\frac{c_{2 i} \Omega_{0}}{c_{3 i} P}\left|\hat{h} r s_{i}\right|^{2}, & w=\frac{c_{1 i} \Omega_{0}}{c_{3 i} P}\left|\hat{h} s_{i} r\right|^{2} \\
\delta=\frac{\Omega_{0}}{P}, & h(\delta)=\frac{\left(8^{v_{j}}-1\right) c_{1 i} c_{2 i}}{P^{2} c_{3 i}} \frac{\Omega_{0}}{P}
\end{array}
$$

Then we have

$$
\operatorname{Pr}\left(\mathcal{I}_{s_{j} s_{i}}^{o}<v_{j}\right)=\operatorname{Pr}\left(x+\frac{u v}{u+v+w+\delta}<h(\delta)\right)
$$

Lemma 1 Let $x$ be an exponential random variable with mean $1 / \lambda_{x}$, $\delta$ be a small positive number, and $h(\delta)$ be a continuous function that $h(\delta) \rightarrow 0$ as $\delta \rightarrow 0$. Let $r_{\delta}$ be a multi-variate function that is independent of variable $x$, and it satisfies the condition that $\lim _{\delta \rightarrow 0} \frac{\operatorname{Pr}\left[r_{\delta}<h(\delta)\right]}{h(\delta)}=A$, where $A$ is a positive constant. Then

$$
\lim _{\delta \rightarrow 0} \frac{1}{h^{2}(\delta)} \operatorname{Pr}\left[x+r_{\delta}<h(\delta)\right]=\frac{A \lambda_{x}}{2}
$$


Lemma 1.3 in [7] is a special form of Lemma 1, in which $r_{\delta}=\frac{y z}{y+z+\delta}$, with $y$ and $z$ being two independent exponentially-distributed random variables. We observe that Lemma 1 holds even if the constraint on $r_{\delta}$ in Lemma 1.3 is relaxed and the proof of Lemma 1 is the same as Lemma 1.3 in [7], and will not be repeated here. With Lemma 1 and Theorem 1, probability $\mathcal{P}_{\text {out }}^{o}$ is derived in $(20)$.

\section{Simulation Results}

Consider a two-way network topology where the distance between two sources is $100 \mathrm{~m}$, and the relay node is located on the line segment between two source nodes. We assume that the distance from source $S_{1}$ to the relay node is $L \mathrm{~m}$, and thus the distance from source $S_{2}$ to the relay is $(100-L) \mathrm{m}$. The signal fading follows the quasi-static Rayleigh distribution and the average channel gain equals $d^{-\beta[8]}$, where $d$ denotes the distance from transmitter to receiver and $\beta$ is the path loss index. Let the path loss index $\beta$ be 3 and the additive noise power $\Omega_{0}$ be $-70 \mathrm{dbm}$. We also assume that ratio $\frac{\Omega_{\hat{h}, a b}}{\Omega_{h, a b}}=0.995$ for transmitter $a$ and receiver $b$ where $a \in\left\{S_{1}, S_{2}, R\right\}$ and $b \in\left\{S_{1}, S_{2}, R\right\}$ in this channel.

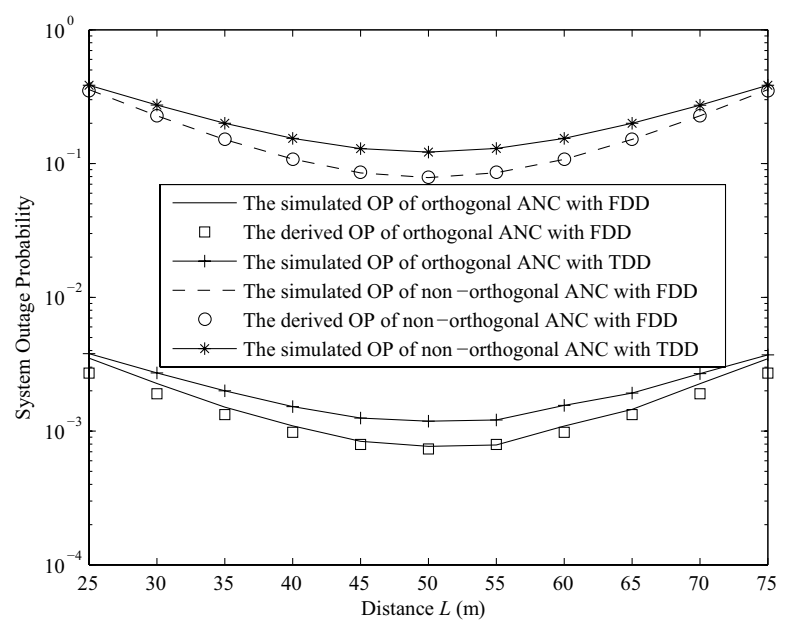

Figure 2 OP versus distance $L$ for non-orthogonal and orthogonal ANC, where $P=20 \mathrm{dbm}$ and $v_{1}=v_{2}=0.5 \mathrm{bits} / \mathrm{Sec} / \mathrm{Hz}$

Figure 2 shows the system outage performance (OP) versus distance $L$ for non-orthogonal and orthogonal ANC, where $P=20 \mathrm{dbm}$ and $v_{1}=v_{2}=0.5 \mathrm{bits} / \mathrm{Sec} / \mathrm{Hz}$. It is shown that the derived OP for the two protocols match the simulated results, which validates the accuracy of our derived expressions. We also observe that orthogonal ANC outperforms non-orthogonal ANC due to higher diversity gain by using the link between source $S_{i}$ to $S_{j}$, and FDD system achieves lower OP than TDD for both the protocols. Figure 3 shows OP versus transmission power $P$ in different transmission-rate scenarios, where $L=50 \mathrm{~m}$. Our derived curves are in agreement with the Monte Carlo simulation results. It is feasible to achieve the lower OP with smaller transmission rates. We also observe that OP reaches a fixed level, called error floor, due to the imperfection in the channel estimation. 


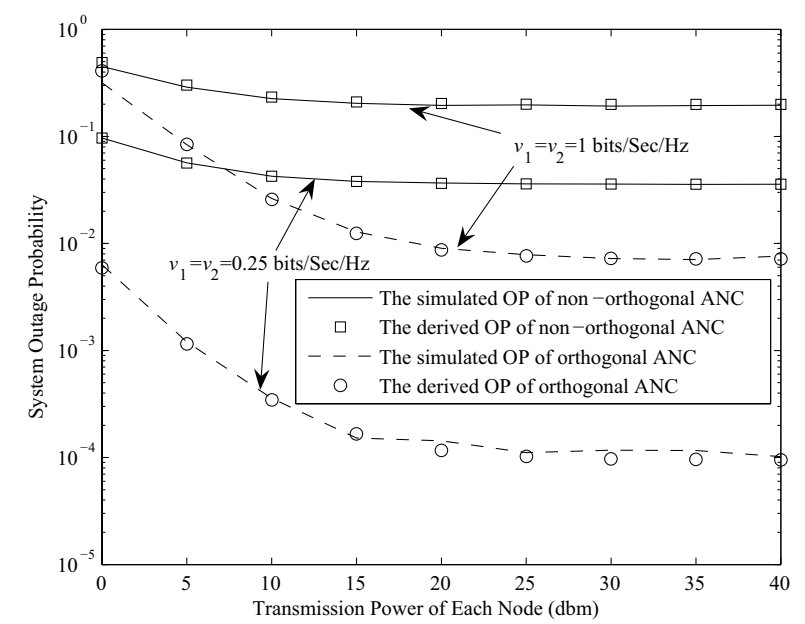

Figure 3 OP versus transmission power $P$ in different transmission-rate scenarios, where $L=50 \mathrm{~m}$

\section{Conclusion}

We have investigated the impact of imperfect CSI on the performance of non-orthogonal and orthogonal ANC in the FDD two way channel. The closed-formed expressions of outage probabilities achieved by non-orthogonal and orthogonal ANC have been derived in the high SNR regime. By using Monte Carlo simulation, it validates that the simulation results are in agreement with the derived results, and FDD system outperforms TDD in terms of OP.

\section{References}

[1] Katti S, Gollakota S, Katabi D. Embracing wireless interference: Analog network coding, ACM Sigcom, Kyoto Japan, Aug 2007.

[2] Ding Z, Leung K, Goeckol D, et al. On the study of network coding with diversity. IEEE Transactions on Wireless Communications, 2009, 8: 1247-1259.

[3] Wang L, Cai Y M, Yang W W. On the finite-SNR DMT of twoway AF relaying with imperfect CSI. IEEE Wireless Commumnications Letters, 2012, 1(3): 161-164.

[4] Wu Y, Patzold M. Outage probability and power allocation of twoway amplify-and-forward relaying with channel estimation errors. IEEE Transactions on Wireless Communications, 2012, 11(6): 1985-1990.

[5] Wang C Y, Lui T C K, Dong X D. Impact of channel estimation error on the performance of amplify-andforward two-way relaying. IEEE Transactions on Vehicular Technology, 2012, 61(3): 1197-1207.

[6] Zhan A, He C, Jiang L G. A channel statistic based power allocation in a butterfly wireless network with network coding. Workshop on CoConet IEEE ICC'10, Cape Town, South Africa, 2010.

[7] Avestimehr A, Tse D N C. Outage capacity of the fading relay channel in the low-SNR regime. IEEE Transactions on Inform Theory, 2007, 53(4): 1401-1415.

[8] Chen C J and Wang L C. Enhancing coverage and capacity for multiuser MIMO system by utilizing scheduling. IEEE Transactions on Wireless Communications, 2006, 5(5): 1148-1157.

[9] Gradshteyn I S, Ryzhik I M. Table of integrals, series and products. 6th ed. Academic Press, 2000. 